\title{
Codeposition of ultrasmooth and high quantum efficiency cesium telluride photocathodes
}

\author{
M. Gaowei, ${ }^{1,}{ }^{*}$ J. Sinsheimer, ${ }^{1}$ D. Strom, ${ }^{2}$ J. Xie, ${ }^{3}$ J. Cen, ${ }^{1}$ J. Walsh, ${ }^{1}$ E. Muller, ${ }^{4}$ and J. Smedley ${ }^{1, \dagger}$ \\ ${ }^{1}$ Brookhaven National Laboratory, Upton, New York 11973, USA \\ ${ }^{2}$ Max Planck Institute for Physics, Munich, 80805, Germany \\ ${ }^{3}$ Argonne National Laboratory, Lemont, Illinois 60439, USA \\ ${ }^{4}$ Stony Brook University, Stony Brook, New York 11794, USA
}

(Received 13 May 2019; published 23 July 2019)

\begin{abstract}
Cesium telluride (CsTe) photocathodes have been the primary choice for electron sources by worldwide accelerators, due to their high quantum yield, stable performance in complex operation environments and long lifetime. In this paper we compared the traditional sequential and the newly developed coevaporation growth of CsTe photocathodes by describing the chemical and structural evolution of each growth method, using in situ, real time x-ray characterization. From the codeposition method, we were able to achieve $\sim 2 \mathrm{~nm}$ surface roughness, high crystallinity and a quantum efficiency of $19 \%$ at $266 \mathrm{~nm}$ wavelength.
\end{abstract}

DOI: 10.1103/PhysRevAccelBeams.22.073401

\section{INTRODUCTION}

For the past decades, cesium telluride (CsTe) has been chosen as the electron source material for high bunch charge, high repetition rate superconducting radio frequency electron injectors, which is essential for the stateof-art energy recovery linacs and high-power free electron lasers (FELs) [1-4]. The application of the cesium telluride photocathode has been reported by accelerators all over the world [5-10]. The popularity of CsTe as cathode material comes from its balanced properties between the high quantum efficiency (over 10\% at its working wavelength), long lifetime (over a span of several months) and its robustness to chemical contamination and high gradient environment compared to other regularly used cathode materials such as GaAs: Cs and multialkali antimonide photocathodes [11]. Recently, the cesium telluride coating was developed to protect the GaAs photocathode as spin-polarized electron sources [12]. The growth recipe of cesium telluride has been studied by various facilities and the conditions and performance vary by quite a bit, indicating the complexity of the mechanisms in the chemical reaction of cesium and tellurium [13]. Efforts of characterizing this mechanism using x-ray photoemission spectroscopy and Auger electron spectroscopy, come

\footnotetext{
*mgaowei@bnl.gov

†Present address: Los Alamos National Laboratory, New Mexico 87545, USA.

Published by the American Physical Society under the terms of the Creative Commons Attribution 4.0 International license. Further distribution of this work must maintain attribution to the author(s) and the published article's title, journal citation, and DOI.
}

to the conclusion that the final product can be a random combination of various Cs-Te compounds, resulting in the differed performance in quantum efficiency and cathode lifetime $[14,15]$. However, compared to the well-developed growth procedure and frequently reported performance study, there still lacks structural and chemical characterization of this material in the community.

In this paper, we present the results from the comparison of the cesium telluride photocathode material prepared by the sequential growth method and codeposition method. Using the codeposition method, we were able to grow CsTe cathode with ultrasmooth surface (roughness $\sim 2 \mathrm{~nm}$ ), high quantum efficiency $(19 \%$ at $266 \mathrm{~nm})$ and highly crystallized structure. Real time in situ $\mathrm{x}$-ray techniques including $\mathrm{x}$-ray fluorescence spectroscopy (XRF), x-ray diffraction (XRD) and X-ray reflectivity (XRR) were used to characterize both growth processes and provide detailed postanalysis. Results from these techniques along with the spectral response are reported.

\section{EXPERIMENTAL}

Cesium telluride photocathodes were synthesized in a custom-built ultrahigh vacuum chamber with tellurium evaporator and Cs effusion cell attached. One gram of Cs breakseal ampoule was loaded into its vacuum housing, with a J-shaped stainless-steel bend attached to the bottom of the housing. This effusion cell was separated with the main deposition chamber by a UHV needle valve. The vacuum housing was preheated, and the glass ampule was broken by crushing from the side. The melted Cs was then collected by a J-shaped stainless-steel tube (J-bend) underneath, which would serve as the Cs reservoir in this experiment. During deposition, the J-bend along with the whole effusion cell 


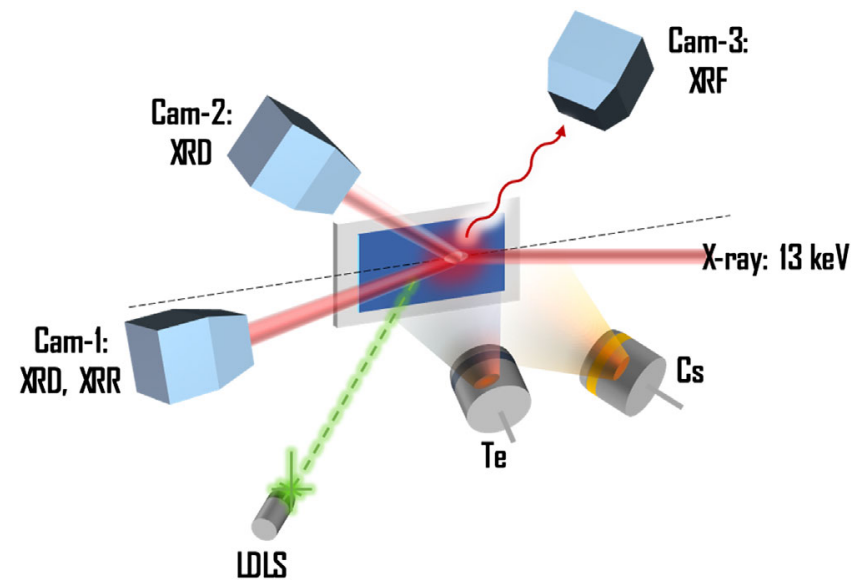

FIG. 1. Schematic diagram of the in situ and real time x-ray characterization setup at beam line G3, CHESS.

vacuum housing were heated and Cs vapor was guided through a stainless-steel pathway heated by circulating hot $\mathrm{N}_{2}$. A shutter was designed and installed in the vacuum chamber at the output of the guiding tube to terminate the Cs vapor flow at the end of the growth when the sources are cooling. The effusion cell output was mounted approximately $45^{\circ}$ below the sample rotation plane and $\sim 10 \mathrm{~cm}$ away from the substrate. The Te evaporator was mounted at the same height with the substrate and when in position, the distance between the Te source and the substrate was $\sim 6 \mathrm{~cm}$. Te was evaporated by heating an alumina crucible loaded with crushed Te metal chunks. The position of the effusion cell and the Te evaporator are illustrated in Fig. 1.

The real time in situ x-ray studies were performed at the Cornell High Energy Synchrotron Source (CHESS) beam line G3. The beam line setup of this experiment is illustrated in Fig. 1. The photon energy of the incident X-ray beam was $13 \mathrm{keV}(\lambda=0.95 \AA)$. The XRR and XRD data were measured using a four-axis diffractometer with two Pilatus $100 \mathrm{~K}$ x-ray cameras. Cam-1 was mounted $100 \mathrm{~cm}$ downstream from the substrate, and Cam-2 was mounted $30^{\circ}$ with Cam- 1 and $30 \mathrm{~cm}$ away from the sample (labeled in Fig. 1). XRR measurements were performed by scanning the $2 \theta$ angle from $0^{\circ}$ to $6^{\circ}$ and XRD measured with a $2 \theta$ range from $5^{\circ}$ to $30^{\circ}$. The XRF spectra were measured by a vortex multicathode $\mathrm{x}$-ray detector mounted $45^{\circ}$ with respect to the sample surface normal and approximately $25 \mathrm{~cm}$ away from the sample.

$\mathrm{Si}$ (100) substrates were freshly etched by hydrofluoric acid to remove the native oxides. Substrates were loaded into the growth chamber and annealed at $550{ }^{\circ} \mathrm{C}$ for $1 \mathrm{hr}$. The evaporation rate was controlled by adjusting the current of evaporators and was measured with a quartz crystal microbalance (QCM) placed alongside the sample. During deposition, the substrate temperature was set to $120^{\circ} \mathrm{C}$, and the growth rate was around $0.1-0.2 \AA / \mathrm{s}$. After growth, the spectral response of each sample from 200 to $400 \mathrm{~nm}$ was measured using an optical system consisting

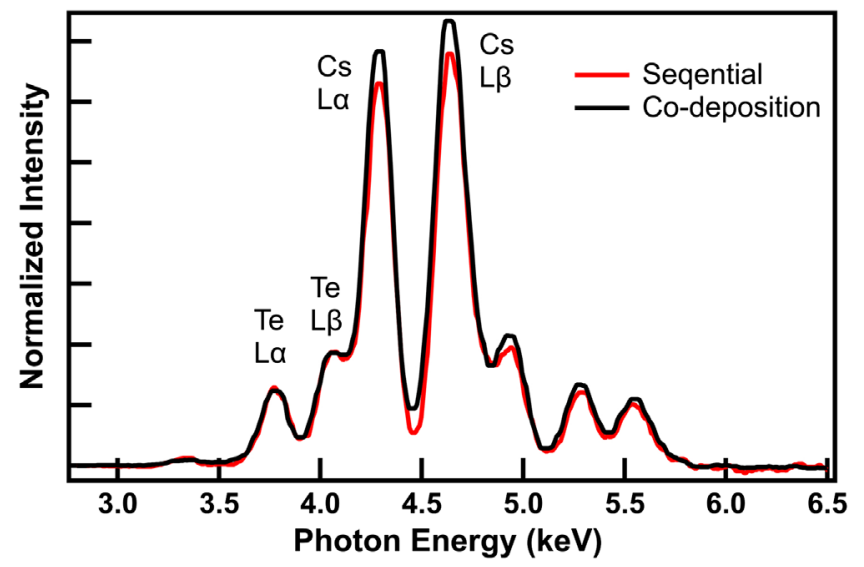

FIG. 2. XRF spectra of sequentially grown (red curve) and codeposited (dark curve) CsTe photocathodes. Curves are normalized with Te peaks. The codeposited cathode incorporates more Cs than the sequential one.

of a laser driven light source and a Cornerstone monochromator. The photocurrent was collected using a Keithley 6517B electrometer.

\section{RESULTS AND DISCUSSION}

$\mathrm{X}$-ray fluorescence spectrum of the 2 CsTe photocathodes grown by sequential and co-dep methods respectively are shown in Fig. 2. Both spectra are normalized with Te $\mathrm{L}$ peaks. The difference in the Cs peak height indicates that the co-dep sample (dark curve) incorporates more Cs during growth than the sequential sample. The XRF spectra were analyzed using the software package PYMCA [16]. The calculated stoichiometry from fitting the spectra is listed in Table I. The fitted stoichiometry for both sequential and co-dep sample is found to be much lower than the believed $\mathrm{Cs}_{2} \mathrm{Te}$.

The evolution of the diffraction pattern from both the sequential growth and the co-dep is shown in Figs. 3 and 4. In Fig. 3, the diffraction peaks of Te appeared around a deposited thickness of $2 \mathrm{~nm}$. The intensity of the diffraction peaks continued to increase while maintaining the same phase throughout the growth. The accumulated thickness of Te deposition on the QCM is $23.5 \mathrm{~nm}$, with a rate controlled at $0.05-0.07 \AA / \mathrm{s}$. Once the Cs deposition started, the Te lines quickly disappeared. This process happened fast and was not captured in our scan. We do observe a chaotic period of growth in the first $10 \mathrm{~nm}$ of Cs deposition, shown in Fig. 3(c). In this period, at least two

TABLE I. Fitted atomic concentration of sequentially and codeposited CsTe photocathodes.

\begin{tabular}{lcc}
\hline \hline Sample & Te & Cs $( \pm 0.05)$ \\
\hline Sequential & 1.00 & 1.15 \\
Codeposition & 1.00 & 1.30 \\
\hline \hline
\end{tabular}


crystal phases appeared and disappeared, marked with dotted lines in Fig. 3(c). and then a stable set of diffraction peaks appeared and became more and more intense as Cs deposition continues. In our study, we do not monitor the QE during growth due to the $\mathrm{x}$-ray induced current that overwhelms the current produced from the UV light. Instead, we monitor the real time $\mathrm{x}$-ray fluorescence signal and stop the growth when we see the Cs peak intensities reach a maximum. At the end of the Cs deposition, we accumulated $150 \mathrm{~nm}$ of Cs on the QCM. The amount of Cs matches the reported Cs thickness from the sequential growth recipe [14]. The fine $2 \theta$ scan after each layer is shown in Fig. 5(a), where the dark line is the diffraction peaks for the Te layer and the red curve is the final CsTe layer. From Fig. 5(a) we can see that after the Cs deposition, almost all the Te peaks are gone, indicating a full conversion of Te material. The weak intensity of the $2 \theta$ scan indicated that the films are not well crystalized. We identified two phases in the final film of the sequentially grown CsTe photocathodes, namely $\mathrm{Cs}_{2} \mathrm{Te}[(203)$ at $d=$ $3.005 \AA,(103)$ at $d=3.60 \AA$ and (111) at $d=4.60 \AA]$ and $\mathrm{Cs}_{2} \mathrm{Te}_{3}$ [(132) at $d=2.85 \AA$, (131) at $d=3.44 \AA$ and
(021) at $d=5.08 \AA$ ] [17]. The combination of the two materials could have resulted in the ratio obtained from the fitting result of the $\mathrm{x}$-ray fluorescence.

The structural evolution of the co-dep sample is much more straightforward. In Fig. 4(b), we can see much fewer diffraction than the sequentially grown CsTe layer. There was only one stable phase throughout the growth. The post growth $2 \theta$ scan is shown in Fig. 5(b). Compared to the sequentially grown layer, the intensities of the diffraction peaks are 2 orders of magnitude higher, indicating a strong crystallinity of this cathode. The two diffraction peaks are identified as the $222(d=2.315 \AA)$ and $111(d=4.615 \AA)$ phase of $\mathrm{Cs}_{2} \mathrm{Te}$ [17]. The diffraction spectrum of this sample is dominated by the $\mathrm{Cs}_{2} \mathrm{Te}$ phase, and no other diffraction peaks were observed. However, the x-ray fluorescence indicates that the film might still have phases that contain a lower Cs to Te ratio. Given that in the sequentially grown film there exist phases that are not well crystalized, it is plausible to believe that there might be other CsTe phases that cannot be detected by diffraction. It was also observed in the image on both cameras (data not

(a)

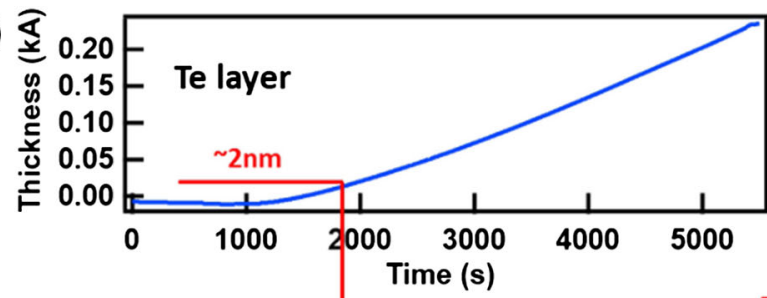

(b)

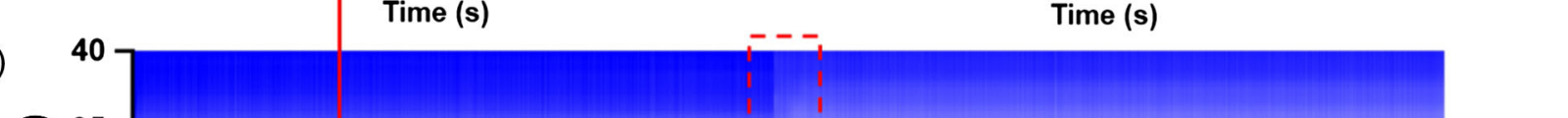

(c)
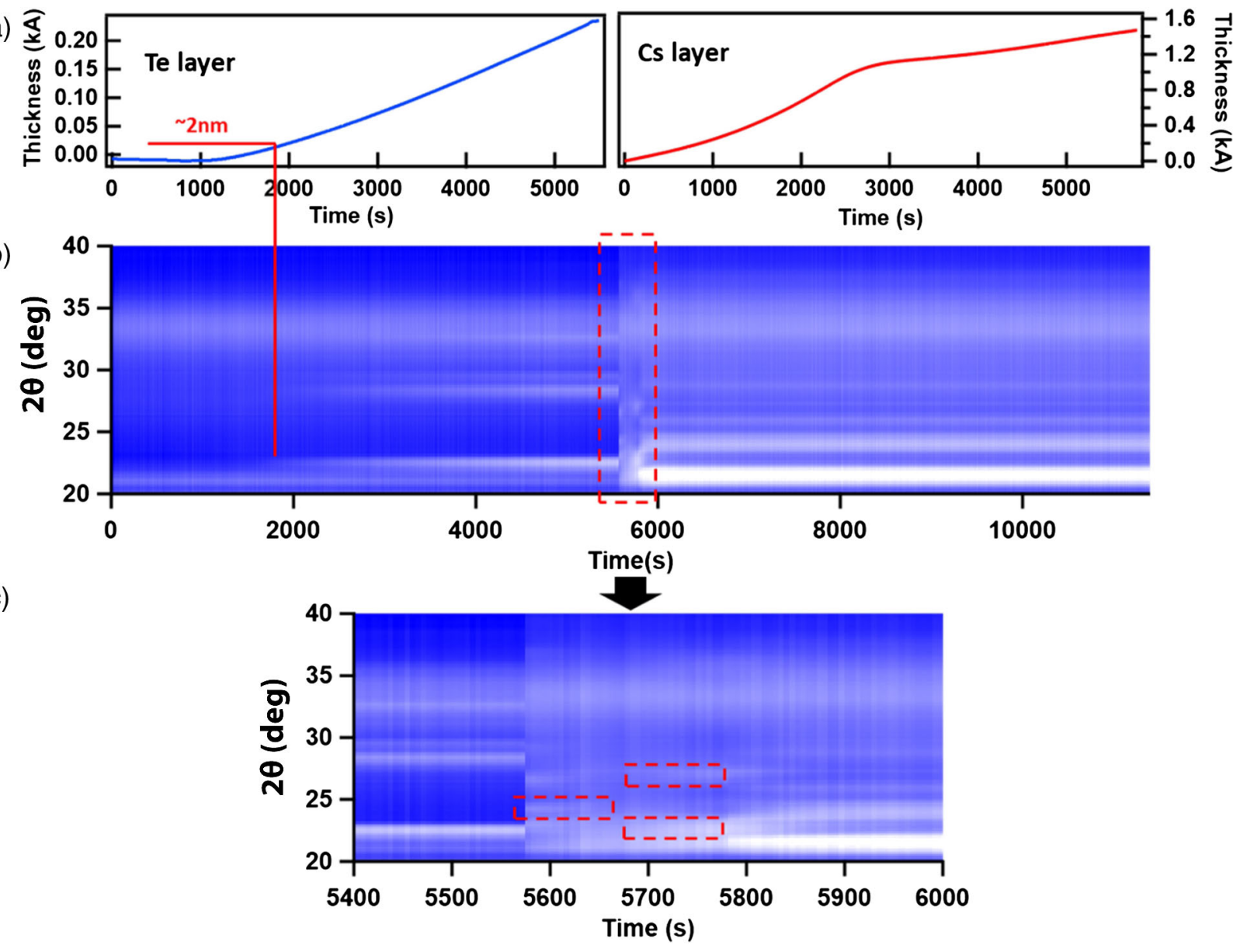

FIG. 3. Structural evolution of a sequentially grown CsTe photocathode with plots showing (a) the thickness increases of each deposition step recorded by the QCM; (b) real time out-of-plane x-ray diffraction pattern of the full growth with the beginning period of Cs step marked in red dotted lines; (c) the marked period zoomed in, showing detailed phase change during the growth. 
(a)

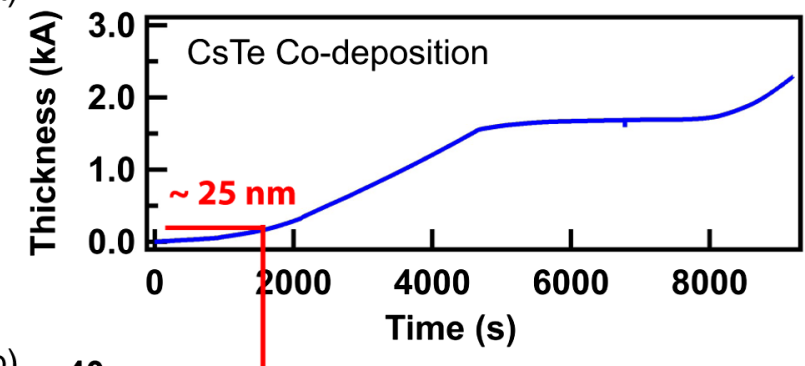

(b)

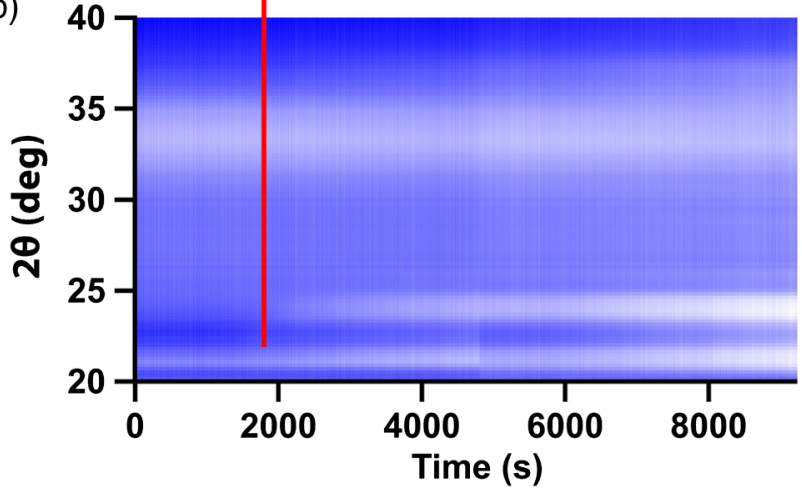

FIG. 4. Structural evolution of a codeposited CsTe photocathode with plots showing (a) the thickness evolution from the QCM and (b) the real time XRD pattern recorded over the entire growth process.

shown) that the diffraction patterns from the sequentially grown layer were continuous lines, while those from the co-dep sample became bright dots, indicating the formation of a strongly textured film. The favorable orientation in this case is (222) for the co-dep sample.

The comparison of the spectral response is shown in Fig. 6. We have repeatedly tested this recipe and got similar QE performance (data not shown). We can see that in the measured range of 250 to $350 \mathrm{~nm}$, the co-dep cathode performs significantly better than the sequential cathode. At the wavelength of $266 \mathrm{~nm}$, which is a typical operation wavelength in many facilities, the co-dep CsTe photocathode reached a QE of $19 \%$, while the sequentially grown cathode is $8.5 \%$, which is similar to the previously reported $\mathrm{QE}[2,5,8,10]$. After depositing excess Cs, the QE of the co-dep sample dropped dramatically, almost similar to the sequentially grown cathode, which might suggest that sequential cathode has excess $\mathrm{Cs}$ at the cathode surface [18]. After exposing the cathode to an unbaked section of the chamber with a $10^{-8}$ torr vacuum, the $\mathrm{QE}$ of the co-dep sample came back to relatively the same $\mathrm{QE}$ as right after the growth, with a slightly better performance in the lower energy range around $300 \mathrm{~nm}$. The change in the behavior of the cathode can be explained by two mechanisms: the removal of the excess Cs and the formation of Cs-O dipole on the cathode surface which lowers the electron affinity, similar to the activation mechanism of GaAs: Cs photocathode [19]. Post-X-ray fluorescence of the co-dep cathode after each treatment did not show an observable difference (a)

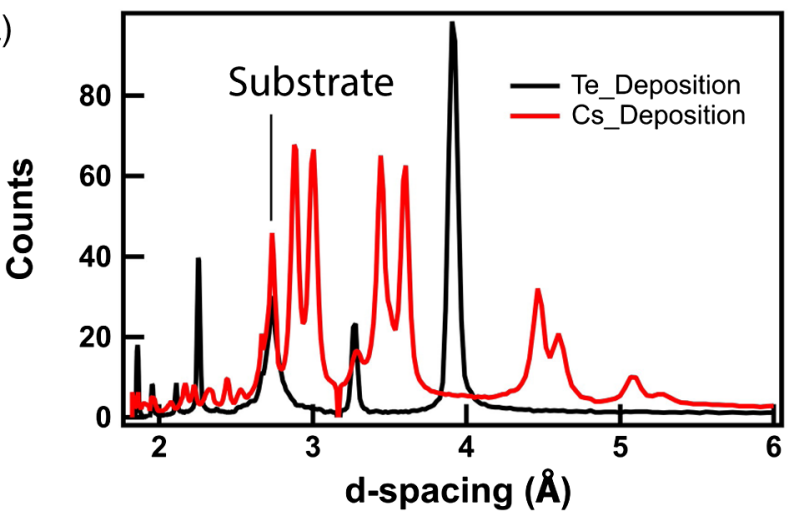

(b)

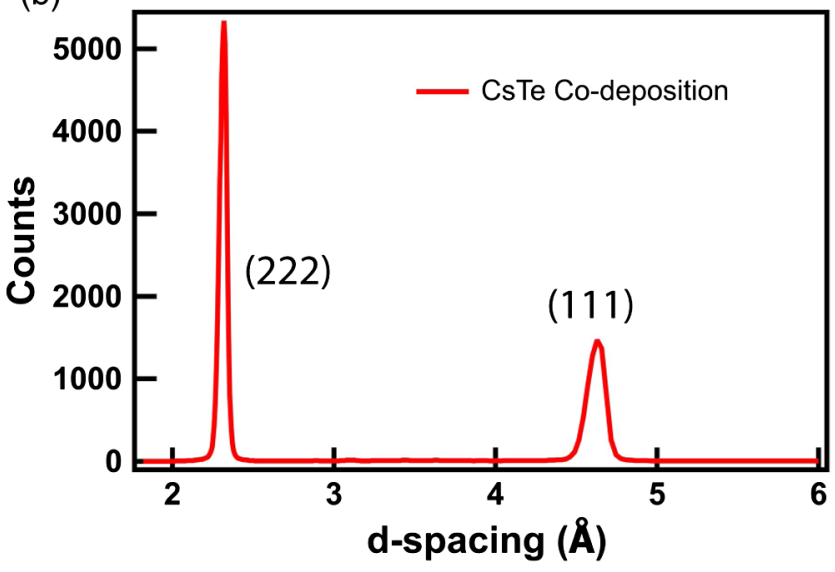

FIG. 5. The XRD spectrum after growth for (a) the sequential deposited and (b) the codeposited CsTe photocathodes.

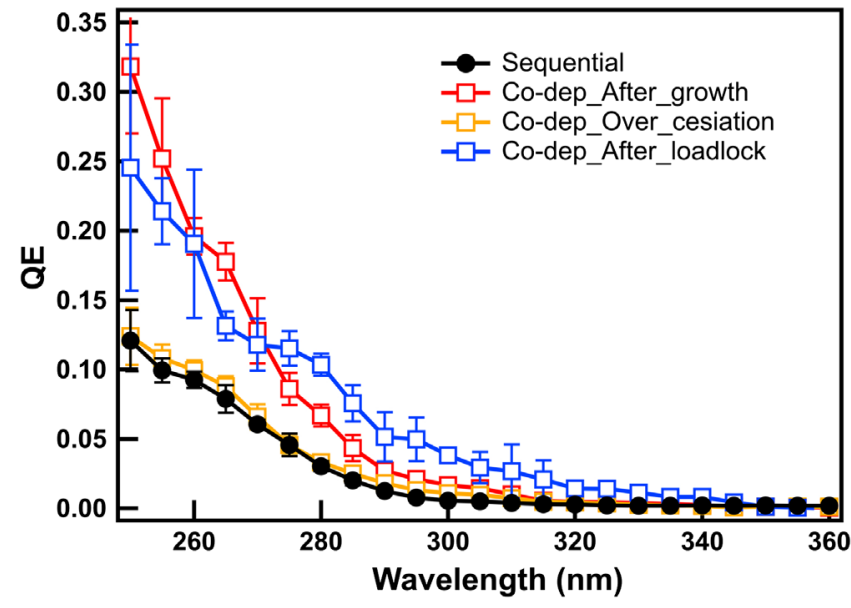

FIG. 6. Comparison of quantum efficiencies of the sequentially grown (solid circles) and codeposited (hollow squares) CsTe photocathodes. After depositing excess Cs, the QE of the co-dep sample dropped dramatically (yellow curve). After exposing the cathode to an unbaked section of the chamber with a $10^{-8}$ torr vacuum, the $\mathrm{QE}$ of the co-dep sample came back to relatively the same QE as right after the growth, with a slightly better performance in the lower energy range around $300 \mathrm{~nm}$ (blue curve). 

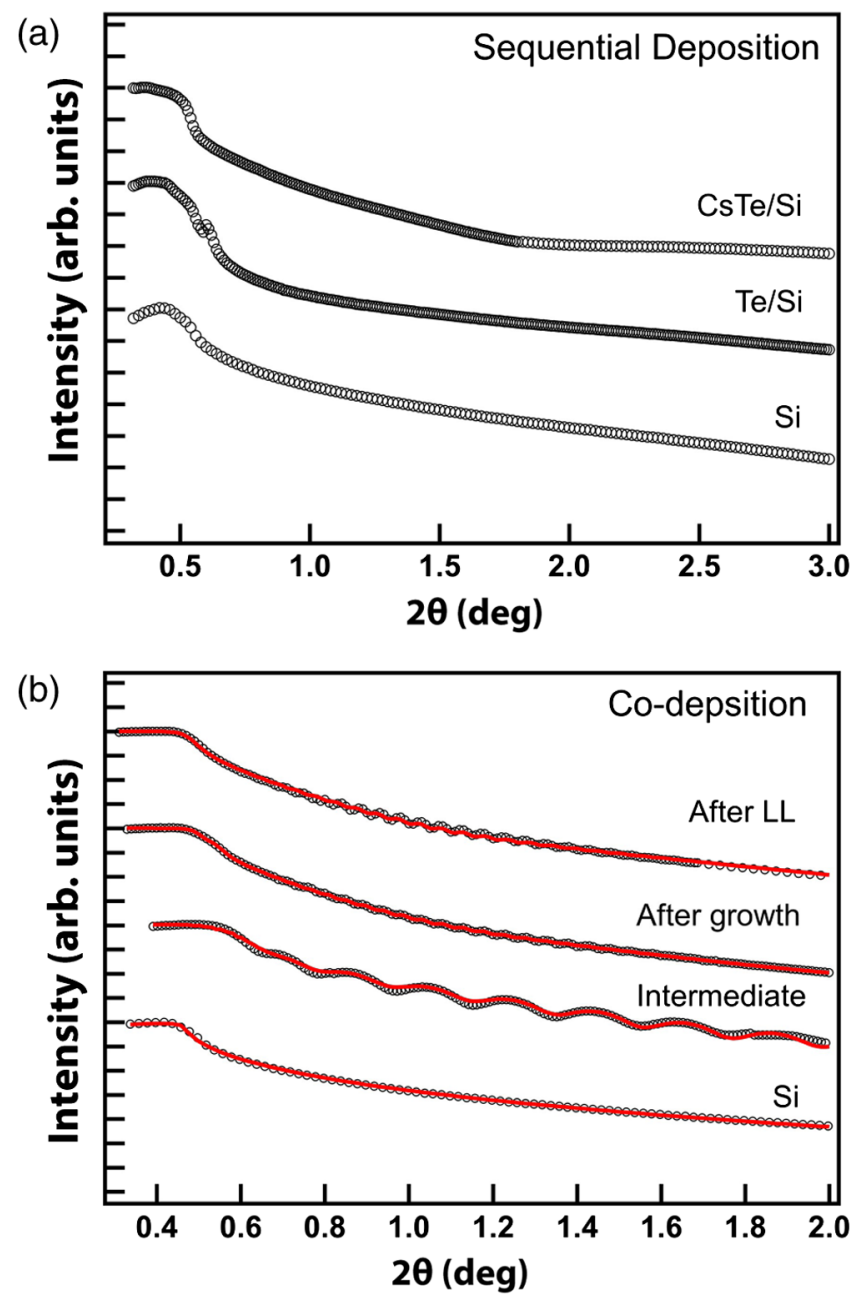

FIG. 7. Experimental data (circles) and fitting (solid line) of XRR profiles after each growth step for (a) sequential and (b) codeposited CsTe photocathodes. The plots are offset for clarity. The sequential deposition films are too rough to make a reliable fitting. The final cathode thickness of codeposited photocathode was fitted to be of $\sim 960 \AA$ (top curve). The oscillations from the $\mathrm{CsTe} / \mathrm{Si}$ sample indicate a relative smooth film surface roughness.

in the Cs composition (data not shown), indicating the mere surface modification of the treatment.

$\mathrm{X}$-ray reflectivity measurements were performed after each deposition step for both the sequential and co-dep samples and shown in Fig. 7. The experimental XRR data of sequential growth sample show no obvious oscillations, indicating thin films with rough surfaces. It is hard for a reliable fitting of these data to estimate the film thickness and surface roughness. On the other hand, the experimental XRR data of the co-dep sample show clear oscillations and fitted with theoretical curves calculated based on layer structure models following Parratt's recursion method [20] to extract film thickness, surface and interface roughness [21]. Figure 7 and Table II lists the fitting results of the $\mathrm{Si}$ substrate, an intermediate layer of $\sim 25 \mathrm{~nm}$, the layer right
TABLE II. XRR fitting results of the codeposited CsTe photocathode.

\begin{tabular}{lcc}
\hline \hline & Thickness $(\AA)$ & Roughness $(\AA)$ \\
\hline After load lock & $968.3 \pm 2.9\left(\right.$ total $\left.\mathrm{Cs}_{2} \mathrm{Te}\right)$ & $19.1 \pm 0.2$ \\
After growth & $1026.1 \pm 1.6\left(\right.$ total $\left.\mathrm{Cs}_{2} \mathrm{Te}\right)$ & $19.10 \pm 0.07$ \\
Intermediate layer & $245.5 \pm 1.7$ & $9.55 \pm 0.14$ \\
Si substrate & $\cdots$ & $3.75 \pm 0.02$ \\
\hline \hline
\end{tabular}

after growth and the final cathode after the removal of excess Cs. The final thickness of cathode is around $100 \mathrm{~nm}$ with a roughness of $\sim 2 \mathrm{~nm}$. After the Cs removal step, the total thickness might have decreased for a few nm with the surface roughness unchanged. The comparison in XRR results indicates a clear improvement in the film roughness for the codeposited CsTe film, which is also observed for alkali antimonide photocathodes [22,23]. In an accelerating field where physical roughness dominates the emittance of the electron beam, a smoother cathode will certainly reduce the field emission and improve the intrinsic emittance of the electron beam from the photocathode $[23,24]$.

\section{SUMMARY}

In summary, we presented results of real time structural analysis and in situ XRF, XRR and spectral response measurements of both sequentially grown and codeposited CsTe photocathodes. The cathodes from our deposition procedure yield a stoichiometry that is less than Cs: $\mathrm{Te}=$ $2: 1$. The comparison between the sequential and co-dep method indicates that the codeposited cathode incorporates more Cs than the sequential ones. CsTe photocathodes grown by the Cs effusion cell and codeposition method result in stable and highly textured crystal structure, with ultrasmooth surface of $2 \mathrm{~nm}$ surface roughness. The QE of the co-dep cathode reached $19 \%$ at $266 \mathrm{~nm}$, while the sequentially grown cathode yields a $\mathrm{QE}$ of $8.5 \%$ at the same wavelength. Excess Cs might result in a decrease of QE and can be recovered by exposing the cathode to slightly higher content of water and oxygen environment.

\section{ACKNOWLEDGMENTS}

The authors would like to acknowledge Arthur Woll, Howie Joress and the beam line staff at Cornell High Energy Synchrotron Source (CHESS), Cornell University, Anna Alexander and Vitaly Pavlenko from Los Alamos National Laboratory for their assistance to the experiment. This work is funded by U.S. Department of Energy, under Contracts No. KC0407-ALSJNT-I0013, No. DE-AC02-98CH1088 and SBIR Grant No. DE-SC0009540. The use of CHESS is supported by the NSF and the NIH/NIGMS under NSF Grants No. DMR-0936384 and No. DMR-1332208. Work at Argonne National Laboratory was supported by the U.S. Department of Energy, Office of Science, Office of High Energy Physics under Contract No. DE-AC02-06CH11357. 
The use of National Synchrotron Light Source II at Brookhaven National Laboratory is supported by U.S. Department of Energy (DOE) Office of Science under Contract No. DE-AC02-98CH10886.

[1] S. H. Kong, D. C. Nguyen, R. L. Sheffield, and B. A. Sherwood, Fabrication and characterization of cesium telluride photocathodes-A promising electron source for the Los-Alamos advanced FEL, Nucl. Instrum. Methods Phys. Res., Sect. A 358, 276 (1995).

[2] D. Filippetto, H. Qian, and F. Sannibale, Cesium telluride cathodes for the next generation of high-average current high-brightness photoinjectors, Appl. Phys. Lett. 107, 042104 (2015).

[3] R. L. Ives, G. Collins, D. Marsden, T. Bui, and E. Montgomery, Long-life photocathodes with high quantum efficiency, in Proceedings of the 18th International Vacuum Electronics Conference (IVEC, London, 2017).

[4] P. Musumeci, J. G. Navarro, J. B. Rosenzweig, L. Cultrera, I. Bazarov, J. Maxson, S. Karkare, and H. Padmore, Advances in bright electron sources, Nucl. Instrum. Methods Phys. Res., Sect. A 907, 209 (2018).

[5] R. Xiang, L. Lin, B. Zhang, X. Lu, S. Quan, L. Wang, and K. Zhao, Research and fabrication of the photocathode used in DC-SC photocathode rf electron gun, At. Energ. Sci. Technol. 38, 185 (2004).

[6] E. E. Wisniewski, D. Velazquez, Z. Yusof, L. Spentzouris, J. Terry, T. J. Sarkar, and K. Harkay, Kelvin probe studies of cesium telluride photocathode for AWA photoinjector, Nucl. Instrum. Methods Phys. Res., Sect. A 711, 60 (2013).

[7] E. Prat, S. Bettoni, H. H. Braun, R. Ganter, and T. Schietinger, Measurements of copper and cesium telluride cathodes in a radio-frequency photoinjector, Phys. Rev. ST Accel. Beams 18, 043401 (2015).

[8] F. Sannibale, M. Zolotorev, R. Wells et al., Status, plans and recent results from the APEX project at LBNL, in Proceedings of the 37th International Free Electron Laser Conference (JACoW, Daejeon, Korea, 2015), pp. 81-84.

[9] R. Corsini, S. Curt, R. Jones et al., First Experiments at the CLEAR User Facility, in Proceedings of the 9th International Particle Accelerator Conference (JACoW, Vancouver, Canada, 2018), pp. 4066-4069.

[10] S. Schreiber, S. Lederer, P. Michelato, L. Monaco, and D. Sertore, Update on the lifetime of $\mathrm{Cs}_{2} \mathrm{Te}$ photocathodes operated at FLASH, in Proceedings of the 38th International Free Electron Laser Conference (JACoW, Geneva, Switzerland, 2018), pp. 407-410.
[11] R. A. Loch, Master thesis, University of Twente, 2005.

[12] J. K. Bae, L. Cultrera, P. DiGiacomo, and I. Bazarov, Rugged spin-polarized electron sources based on negative electron affinity GaAs photocathode with robust $\mathrm{Cs}_{2} \mathrm{Te}$ coating, Appl. Phys. Lett. 112, 154101 (2018).

[13] A. Ruth, K. Nemeth, K. C. Harkay, J. Z. Terdik, L. Spentzouris, and J. Terry, Searching for low-workfunction phases in the Cs-Te system: The case of $\mathrm{Cs}_{2} \mathrm{Te}_{5}$, J. Appl. Phys. 113, 183703 (2013).

[14] A. di Bona, F. Sabary, S. Valeri, P. Michelato, D. Sertore, and G. Suberlucq, Auger and X-ray photoemission spectroscopy study on $\mathrm{Cs}_{2}$ Te photocathodes, J. Appl. Phys. 80, 3024 (1996).

[15] F. Sabary, S. Valeri, P. Michelato, C. Pagani, and D. Sertore, Formation of the $\mathrm{Cs}_{2} \mathrm{Te}$ photocathode: Auger and photoemission spectroscopy study, in Proceedings of the 5th European Particle Accelerator Conference (JACoW, Sitges, Spain, 1996).

[16] V. A. Sole, E. Papillon, M. Cotte, P. Walter, and J. Susini, A multiplatform code for the analysis of energy-dispersive X-ray fluorescence spectra, Spectrochim. Acta B Atom. Spectros. 62, 63 (2007).

[17] A. Jain et al., Commentary: The materials project: A materials genome approach to accelerating materials innovation, APL Mater. 1, 011002 (2013).

[18] S. Schubert, M. Ruiz-Oses, I. Ben-Zvi et al., Bi-alkali antimonide photocathodes for high brightness accelerators, APL Mater. 1, 032119 (2013).

[19] C. Y. Su, W. E. Spicer, and I. Lindau, Photoelectron spectroscopic determination of the structure of $(\mathrm{Cs}, \mathrm{O})$ activated gas (110) surfaces, J. Appl. Phys. 54, 1413 (1983).

[20] L. G. Parratt, Surface studies of solids by total reflection of X-rays, Phys. Rev. 95, 359 (1954).

[21] J. Q. Xie et al., Synchrotron X-ray study of a low roughness and high efficiency $\mathrm{K}_{2} \mathrm{CsSb}$ photocathode during film growth, J. Phys. D 50, 205303 (2017).

[22] Z.H. Ding et al., Temperature-dependent quantum efficiency degradation of K-Cs-Sb bialkali antimonide photocathodes grown by a triple-element codeposition method, Phys. Rev. Accel. Beams 20, 113401 (2017).

[23] J. Feng, S. Karkare, J. Nasiatka, S. Schubert, J. Smedley, and $\mathrm{H}$. Padmore, Near atomically smooth alkali antimonide photocathode thin films, J. Appl. Phys. 121, 044904 (2017).

[24] T. Vecchione, I. Ben-Zvi, D. H. Dowell, J. Feng, T. Rao, J. Smedley, W. Wan, and H. A. Padmore, A low emittance and high efficiency visible light photocathode for high brightness accelerator-based X-ray light sources, Appl. Phys. Lett. 99, 034103 (2011). 